\title{
Effects of Quinidine and Amiodarone on Blood Pressure During Rapid Ventricular Pacing in Coronary Artery Disease
}

\author{
Hugh Calkins, MD, Yu Shyr, MS, Anthony Schork, PhD, Alan Kadish, MD, and Fred Morady, MD
}

$\mathbf{T}$ he hemodynamic response to ventricular tachycardia is an important determinant of prognosis. ${ }^{1}$ Many factors determine the hemodynamic response to ventricular tachycardia, including (1) the rate of the ventricular tachycardia, ${ }^{2,3}$ (2) systolic and diastolic ventricular function, ${ }^{4,5}$ and (3) the neurohumoral response to the arrhythmia. ${ }^{6,7}$ Antiarrhythmic drugs such as quinidine and amiodarone may either improve hemodynamics during ventricular tachycardia by slowing the rate of the tachycardia, or may impair the hemodynamic response to ventricular tachycardia by decreasing ventricular contractility, blunting the neurohumoral response to the tachycardia, or by causing vasodilation..$^{8-14}$ No prior studies have evaluated the effect of antiarrhythmic drugs on the hemodynamic response to ventricular tachycardia independent of their effects on the rate of the tachycardia. The objective of this study was to determine the relative effects of quinidine and amiodarone on the blood pressure (BP) response to rapid ventricular pacing in humans. Previous studies have demonstrated that the BP response during ventricular tachycardia and during ventricular pacing are similar. ${ }^{2}$ Therefore, in this study ventricular pacing was used to assess the effects of antiarrhythmic drugs on the BP response independent of heart rate.

The subjects of this study were 9 patients ( 8 men and 1 woman, mean age $62 \pm 7$ years) who had inducible sustained monomorphic ventricular tachycardia during electrophysiologic testing and who underwent electropharmacologic testing with both quinidine and amiodarone. The indication for electrophysiologic testing was aborted sudden death in 2 patients, syncope in 6 patients, and nonsustained ventricular tachycardia in 1 patient. Each patient had coronary artery disease and a history of a prior myocardial infarction. The mean left ventricular ejection fraction was $0.33 \pm 0.11$. Each of the 9 patients was clinically stable at the time of their initial electrophysiology test and throughout the period of evaluation. No patient had anginal symptoms, a history of a myocardial infarction within the prior 3 months, or congestive heart failure requiring adjustment of their medical regimen. Six patients were being treated with digoxin, 5 with diuretics, 4 with an angiotensin-converting enzyme inhibitor, and 1 patient with a calcium antagonist. The dose of calcium antagonists and angiotensinconverting enzyme inhibitors was kept constant throughout the period of evaluation. The dose of digoxin was reduced by $50 \%$ at the time of initiation of quinidine and amiodarone therapy.

Electrophysiology tests were performed in the fasting state $\geq 5$ half-lives after discontinuation of all antiar-

From the Division of Cardiology, University of Michigan Medical Center, B1 F245, 1500 East Medical Center Drive, Ann Arbor, Michigan 48109-0022; and the Department of Biostatistics, School of Public Health, Ann Arbor, Michigan. Manuscript received March 9, 1992; revised manuscript received and accepted June 4, 1992. rhythmic drugs and $\beta$ blockers. Each patient gave informed consent. Quadripolar electrode catheters were inserted into a femoral vein and positioned at the high right atrium, across the tricuspid valve to record a His bundle electrogram, and at the apex of the right ventricle. A 5 Fr cannula was inserted into a femoral artery for $B P$ monitoring. Leads $V_{l}, I$ and III, the intracardiac electrograms, and BP were recorded at a paper speed of 10 or $25 \mathrm{~mm} / \mathrm{s}$ using a Siemans-Elema Mingograph 7 recorder.

All patients also underwent electrophysiologic testing after treatment with quinidine and amiodarone. Follow-up testing during quinidine therapy was performed $\geq 48$ hours after initiation of treatment with quinidine gluconate at a dose of 486 to $648 \mathrm{mg} 3$ times a day. The follow-up electrophysiology test was performed $<2$ hours before the next scheduled dose. The mean plasma quinidine concentration at the time of follow-up electrophysiologic testing was $3.2 \pm 1.0 \mathrm{mg} /$ liter (range 2.2 to $4.3 \mathrm{mg} /$ liter). Each patient still had inducible sustained monomorphic ventricular tachycardia during treatment with quinidine and was then treated with amiodarone. Follow-up electrophysiologic testing during amiodarone therapy was performed 9 to 10 days after initiation of treatment with amiodarone at a dose of $1,800 \mathrm{mg} /$ day in 3 divided doses.

The pacing protocol was performed in each patient after completion of the clinically indicated portion of the electrophysiology test. Pacing was performed at the right ventricular apex at cycle lengths of $600,500,400,350$, 300,280 and $260 \mathrm{~ms}$. Ventricular pacing was performed at each cycle length for 30 to 40 seconds or until the patient became presyncopal. The minimal duration of pacing at each cycle length was 20 seconds. The sequence of cycle lengths at which pacing was performed was randomized. If the patient's systolic BP was $<40 \mathrm{~mm} \mathrm{Hg}$ at a given cycle length, pacing at shorter cycle lengths was not performed. Between pacing at each cycle length, the patient was allowed to recover for $\geq 5$ minutes or until mean BP returned to within $5 \mathrm{~mm} \mathrm{Hg}$ of the baseline mean BP. No patient developed angina or ischemic $S T$ changes during pacing.

At each pacing cycle length the systolic, diastolic and mean BPs were measured after 30 seconds of pacing or at the point at which the patient became presyncopal (minimum of 20 seconds). BP was determined by averaging the BP response during 4 to 6 beats. Thirty seconds was selected because previous studies have demonstrated that the initial abrupt decrease in BP during rapid ventricular pacing is followed by an increase in BP. Stabilization of the BP is generally achieved within 30 seconds of the onset of rapid ventricular pacing. ${ }^{6,7}$ To confirm stabilization of $B P$ within 30 seconds of pacing, $B P$ was compared 30 and 40 seconds after onset of pacing in each patient at 1 or more cycle lengths between 300 and 400 


\begin{tabular}{|c|c|c|c|c|}
\hline Cycle Length (ms) & Baseline & Quinidine & Amiodarone & $p$ Value \\
\hline Sinus rhythm & $138 \pm 28$ & $124 \pm 28$ & $133 \pm 24$ & 0.19 \\
\hline 600 & $128 \pm 27$ & $120 \pm 31$ & $129 \pm 26$ & 0.33 \\
\hline 500 & $131 \pm 26$ & $121 \pm 26$ & $123 \pm 29$ & 0.29 \\
\hline 400 & $120 \pm 17$ & $109 \pm 28$ & $111 \pm 35$ & 0.33 \\
\hline 350 & $97 \pm 18$ & $81 \pm 29$ & $79 \pm 34$ & $0.05^{*}$ \\
\hline 300 & $65 \pm 14$ & $55 \pm 23$ & $56 \pm 28$ & 0.27 \\
\hline 280 & $52 \pm 9$ & $44 \pm 14$ & $42 \pm 15$ & $0.03 \dagger$ \\
\hline 260 & $39 \pm 7$ & $40 \pm 8$ & $39 \pm 16$ & 0.28 \\
\hline \multicolumn{5}{|c|}{$\begin{array}{l}{ }^{*} p=0.1 \text { baseline versus quinidine; } p=0.04 \text { baseline versus amiodarone; } p=0.18 \\
\text { quinidine versus amiodarone. } \\
\quad t p=0.05 \text { baseline versus quinidine; } p=0.04 \text { baseline versus amiodarone, } p= \\
0.18 \text { quinidine versus amiodarone. }\end{array}$} \\
\hline
\end{tabular}

ms. Neither the systolic nor the diastolic BP differed at 30 and 40 seconds of pacing $(p>0.2)$.

The effects of quinidine and amiodarone on BP during ventricular pacing were evaluated in 2 ways. In the first analysis, the systolic and mean BP at each paced cycle length in the baseline state and during therapy with quinidine and amiodarone were compared using a repeated-measures analysis of variance and paired $\mathrm{t}$ tests. To determine the independent effects of cycle length and drug regimen on the BP response to ventricular pacing, $a$ repeated-measures analysis of variance for 2 factors, both of which are repeated, was also performed. The second method of analysis involved modeling the relation between BP and the pacing rate via polynomial regressions, and comparing the estimated coefficients for the model of this relation under the 3 conditions. For each of the 2 variables, mean BP and systolic BP, models relating these variables to heart rate were developed as follows: First, we investigated which regression model linear, quadratic or cubic - adequately fit the data for each patient and condition separately. Based on the coef-

\begin{tabular}{|ccccc|}
\hline \multicolumn{5}{|c|}{ TABLE II Mean Blood Pressure During Ventricular Pacing } \\
\hline Cycle Length (ms) & Baseline & Quinidine & Amiodarone & $p$ Value \\
\hline Sinus rhythm & $89 \pm 13$ & $81 \pm 13$ & $84 \pm 10$ & 0.08 \\
600 & $87 \pm 15$ & $79 \pm 14$ & $86 \pm 13$ & 0.06 \\
500 & $87 \pm 15$ & $80 \pm 12$ & $84 \pm 13$ & 0.19 \\
400 & $84 \pm 13$ & $74 \pm 16$ & $77 \pm 20$ & 0.19 \\
350 & $69 \pm 13$ & $58 \pm 18$ & $56 \pm 21$ & $0.04^{*}$ \\
300 & $50 \pm 7$ & $43 \pm 13$ & $41 \pm 16$ & 0.08 \\
280 & $41 \pm 7$ & $35 \pm 11$ & $36 \pm 9$ & 0.14 \\
260 & $31 \pm 4$ & $28 \pm 6$ & $30 \pm 9$ & 0.24 \\
\hline * $p=0.03$ baseline versus quinidine; $p=0.05$ baseline versus amiodarone; $p=$ \\
0.75 quinicine versus amiodarone. \\
\hline
\end{tabular}

ficients of determination $\left(r^{2}\right)$, it was concluded that a linear model explained a sufficient proportion of the variation for both mean BP and systolic BP (average $r^{2}$ was 0.86 and 0.89 for mean $B P$ and systolic $B P$, respectively). Next, using the estimated intercept and slope from these linear regressions for each patient and condition, a repeated-measures analysis of variance and paired $\mathrm{t}$ tests were computed to assess whether differences among the 3 conditions exist. A p value $<0.05$ was considered significant.

Table I summarizes the effect of pacing on systolic $B P$ in the baseline state and during therapy with quinidine and amiodarone. Systolic BP was no different during quinidine and amiodarone therapy than under baseline conditions at most paced cycle lengths. However, at a paced cycle length of $350 \mathrm{~ms}$, systolic BP was lower during amiodarone therapy than in the baseline state, and at a paced cycle length of $280 \mathrm{~ms}$, systolic BP was lower during quinidine and amiodarone therapy than in the baseline state. An analysis of variance for 2 repeated factors (drug regimen and cycle length) demonstrated that there was a significant independent effect of cycle
FICURE 1. Linear regreasions of syatolice blood preseure on heart rate (pacing) by drue thereples. The prodicted systolic blood precsevere is shown on the vertical axis and the ventricular pacing rate is showm on the horizontel axls. BPM = beats/min.

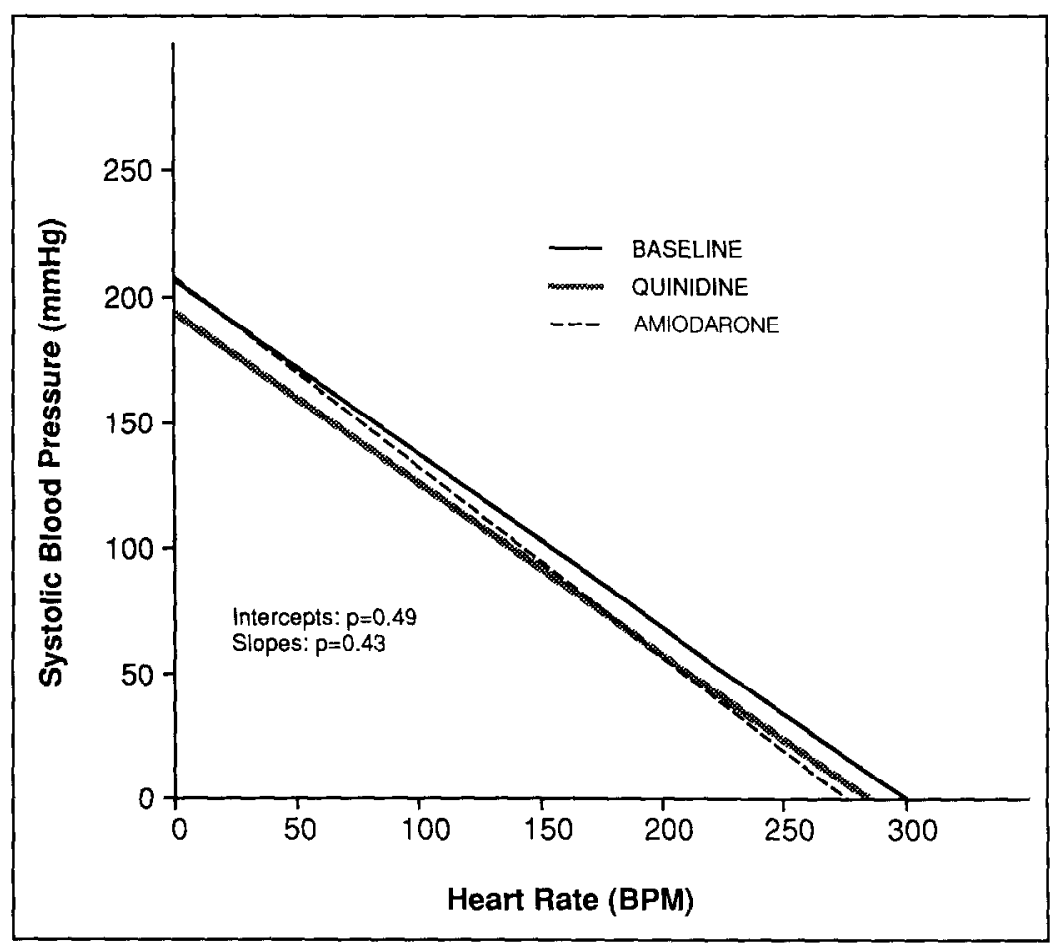


length on the systolic BP response to pacing $(p<0.001)$ but that there was no effect of drug regimen on the systolic BP response to ventricular pacing $(p=0.08)$.

Table II describes the effect of pacing on the mean $B P$. The mean $B P$ was no different during quinidine and amiodarone therapy than under baseline conditions at all paced cycle lengths except at a cycle length of $350 \mathrm{~ms}$. During ventricular pacing at $350 \mathrm{~ms}$, the mean BP was lower during both quinidine and amiodarone therapy than in the baseline state. An analysis of variance for 2 repeated factors (drug regimen and cycle length) demonstrated that there was a significant independent effect of cycle length on the mean BP response to pacing ( $p$ $<0.001)$, but that there was no effect of drug regimen on the mean $B P$ response to ventricular pacing $(p=0.11$ ).

Neither the $y$-intercept nor the slope of the inverse linear relation between heart rate and systolic BP were different in the baseline state than during quinidine and amiodarone therapy (207 \pm 40 vs $194 \pm 45$ vs $208 \pm 41$ $\mathrm{mm} \mathrm{Hg}, p=0.5$ and $-0.7 \pm 0.2 v s-0.7 \pm 0.2 v s-0.8 \pm$ $0.2, p=0.4$, respectively; Figure 1). Similarly, neither the $y$-intercept nor the slope of the inverse linear relation between heart rate and mean $B P$ were different in the baseline state than during quinidine and amiodarone therapy $(133 \pm 26$ vs $120 \pm 21$ vs $132 \pm 18 \mathrm{~mm} \mathrm{Hg}$, $p=0.14$ and $-0.4 \pm 0.1$ vs $-0.4 \pm 0.1$ vs $-0.4 \pm 0.1$, $p=0.09$, respectively; Figure 2).

The main finding of this study is that quinidine and amiodarone do not significantly alter the BP response to rapid ventricular pacing. At almost all paced cycle lengths, systolic and mean BP were no different during therapy with quinidine and amiodarone than in the baseline state. Furthermore, the drug regimen did not have an independent effect on the BP response to pacing, and the slope and $y$-intercept of the linear relation between systolic and mean BP and heart rate were not altered by quinidine and amiodarone therapy. These findings suggest that the predominant mechanism by which quinidine and amiodarone influence BP response to ventricular tachycardia is by altering the rate of the tachycardia.

There are several mechanisms by which quinidine and amiodarone may influence BP response to pacing. First, both amiodarone and quinidine have been shown to impair myocardial contractility in experimental models. ${ }^{9}$ Second, both agents may alter autonomic tone during ventricular tachycardia. Previous studies have demonstrated that recovcry from the initial decrease in BP at the onset of ventricular tachycardia depends on both $\alpha$-adrenergic vasoconstriction and $\beta$-adrenergic augmentation of contraction and relaxation. ${ }^{6}$ Amiodarone, a noncompetitive $\beta$ blocker, may therefore blunt the $\beta$-adrenergic augmentation of contraction and relaxation and quinidine may cause $\alpha$-adrenergic blockade of both venous and arterial vascular beds. ${ }^{10}$ Third, both quinidine and amiodarone have been demonstrated to be direct vasodilators capable of reducing peripheral vascular tone..$^{11,12}$

Despite the presence of several mechanisms by which quinidine and amiodarone may influence hemodynamics, the results of this study demonstrate that quinidine and amiodarone do not have a major effect on the BP response to pacing. At nearly all paced cycle lengths there was no difference in BP during therapy with quinidine and amiodarone compared with the baseline state. Furthermore, the drug regimen did not have an independent effect on BP, and the overall linear relation between pacing rate and $\mathrm{BP}$ was not altered after quinidine and amiodarone therapy. These findings are consistent with the findings of previous clinical studies demonstrating that the hemodynamic effects of quinidine and amiodarone are slight and that these agents generally are well tolerated clinically. . $^{13,14}$

A limitation to this study is that the 3 electrophysiology tests that were performed in the baseline state and during quinidine and amiodarone therapy were per-

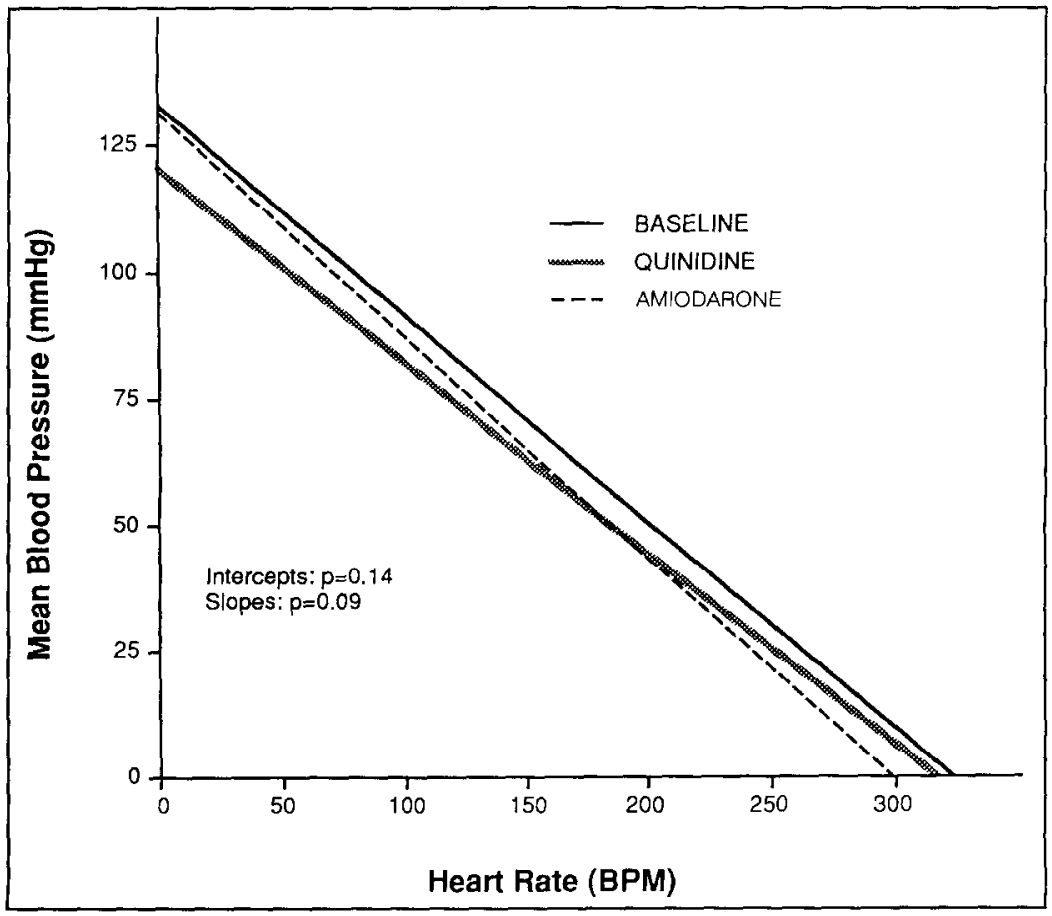

FIGURE 2. Linear regressions of mean blood pressure on heart rate (pacing) by drug therapies. The predicted mean blood pressure is shown on the vertical axis and the ventricular pacing rate is shown on the horizontal axis. BPM = beats/min. 
formed on separate days. Factors related to the duration of hospitalization, e.g., the degree of physical deconditioning or the patient's emotional status, were not controlled and may have influenced BP response to pacing. A second limitation is that a complete evaluation of the patient's hemodynamic status was not performed. Although no difference was found in the BP response to pacing, it is possible that the cardiac output or other hemodynamic variables may have been altered by the antiarrhythmic agents that were studied. A third limitation is that the BP was evaluated while patients were in the supine position. Quinidine or amiodarone may have affected the patient's BP response to rapid ventricular pacing in the upright position.

Potential adverse clinical effects of antiarrhythmic agents include proarrhythmia, and the exacerbation of congestive heart failure. ${ }^{8}$ Recently, several investigators have suggested that antiarrhythmic agents may also have an adverse clinical effect by aggravating the hemodynamic response to ventricular tachycardia ${ }^{15}$ - namely, a patient with hemodynamically stable ventricular tachycardia in the absence of antiarrhythmic therapy may have hemodynamically unstable ventricular tachycardia during antiarrhythmic therapy. The results of this study suggest that this third type of adverse clinical effect is not a prominent factor for quinidine and amiodarone, and suggest that the effect of these antiarrhythmic agents on BP response to ventricular tachycardia results predominantly from their effect on the ventricular tachycardia rate.

1. Waller TJ, Kay HR, Spielman SR, Kutalek SP, Greenspan AM, Horowitz LN. Reduction in sudden death and total mortality by antiarrhythmic therapy evaluated by electrophysiologic drug testing: criteria of efficacy in patients with sustained ventricular tachyarrhythmia. $J$ Am Coll Cardiol 1987;10:83-89. 2. Saksena S, Ciccone JM, Craelius W, Pantopoulos D, Rothbart ST, Werres R. Studies on left ventricular function during sustained ventricular tachycardia. $J$ Am Coll Cardiol 1984;4:501-508.

3. Hamer AWF, Rubin SA, Peter T, Mandel WJ. Factors that predict syncope during ventricular tachycardia in patients. Am Heart $J$ 1984;107:997-1005.

4. Lima JAC, Weiss JL, Guzman PA, Weisfeldt ML, Reid PR, Traill TA. Incomplete filling and incoordinate contraction as mechanisms of hypotension during ventricular tachycardia in man. Circulation 1983;68:928-938.

5. Hamer AW, Zaher CA, Rubin SA, Peter T, Mandel WJ. Hemodynamic benefits of synchronized 1:1 atrial pacing during sustained ventricular tachycardia with severely depressed ventricular function in coronary heart disease. $\mathrm{Am} \mathrm{J}$ Cardiol 1985;55:990-994.

6. Feldman T, Carroll JD, Munkenbeck F, Alibali P, Feldman M, Coggins DL, Gray KR, Bump T. Hemodynamic recovery during simulated ventricular tachycardia: role of adrenergic receptor activation. Am Heart $J$ 1988;115:576589.

7. Crozier IG, Ikram H, Nicholls G. Hemodynamic and hormone changes during induced ventricular tachycardia secondary to coronary artery disease. $\mathrm{Am} J$ Cardiol 1989;63:619-621.

8. Block PJ, Winkle RA. Hemodynamic effects of antiarrhythmic drugs. Am J Cardiol 1983;52:14C-23C

9. Hammermeister KE, Boerth RC, Warbasse JR. The comparative inotropic effects of six clinically used antiarrhythmic agents. Am Heart $J$ 1972;84:643-652. 10. Schmid PG, Nelson LD, Mark AL, Heistad DD, Abboud FM. Inhibition of adrenergic vasoconstriction by quinidine. J Pharmacol Exp Ther 1974;188: 124-134

11. Nelson LD, Schmid PG, Holmsten D, Mark AL, Heistad DD, Abboud FM. Effects of quinidine on venous responses to adrenergic and nonadrenergic constrictor stimuli: indirect evidence of two sites of action in vascular smooth muscle. Proc Soc Exp Biol Med 1974;146:409-413.

12. Cote P, Bourassa MG, Delaye J, Janin A, Froment R, David P. Effccts of amiodarone on cardiac and coronary hemodynamics and on myocardial metabolism in patients with coronary artery disease. Circulation 1979;59:11651172

13. Crawford MH, While DH, O'Rourke RA. Effects of oral quinidine on left ventricular performance in normal subjects and patients with congestive cardiomyopathy. Am J Cardiol 1979;44:714-718.

14. Kaski JC, Girotti LA, Messuti H, Rutitzky B, Rosenbaum MB. Long-term management of sustained, recurrent symptomatic ventricular tachycardia with amiodarone. Circulation 1981;101:759-768.

15. Sharma AD, Purves P, Yee R, Klein G, Jablonsky G, Kostuk WJ. Hemodynamic effects of intravenous procainamide during ventricular tachycardia. $\mathrm{Am}$ Heart $J$ 1990;119;1034-1041.

\title{
Regression of Left Ventricular Hypertrophy in Systemic Hypertension with Beta Blockers (Propranolol, Atenolol, Metoprolol, Pindolol and Celiprolol)
}

\author{
Gregory P. Vyssoulis, MD, Eva A. Karpanou, MD, Christos E. Pitsavos, MD,
} Athanasios A. Paleologos, MD, and Pavlos K. Toutouzas, MD

eft ventricular (LV) hypertrophy is frequently associated with systemic arterial hypertension and is a recognized, independent risk factor for coronary artery disease. ${ }^{1,2}$ Reduction of LV hypertrophy is thus a desir-

From the Hypertension Center, Department of Cardiology, University of Athens, Hippokration Hospital, Athens, Greece. Dr. Vyssoulis' current address is: 17 Panagiotou Str., Papagou, 15669 Athens, Greece. Manuscript received January 27, 1992; revised manuscript received June 12, 1992, and accepted June 13. able goal in the treatment of high blood pressure (BP), but not all antihypertensive drugs accomplish this. ${ }^{3}$ Betaadrenergic blocking agents have not been shown to reverse LV hypertrophy in patients with essential hypertension, but few comparative and quantitative studies with different $\beta$ blockers have been reported. ${ }^{4}$ This report concerns a study of the effects on echocardiographic LV hypertrophy indexes in 145 hypertensive patients treated with 5 different $\beta$-blocking drugs.

\begin{tabular}{|lccccc|}
\hline \multicolumn{6}{|l|}{ TABLE I Characteristics of Patients in Each Treatment Group } \\
\hline & Propranolol & Atenolol & Metroprolol & Pindolol & Celiprolol \\
\hline Age (year) & $51 \pm 9$ & $48 \pm 10$ & $51 \pm 11$ & $51 \pm 12$ & $47 \pm 11$ \\
Patients (men/women) & $23(17 / 6)$ & $21(16 / 5)$ & $18(11 / 7)$ & $35(18 / 17)$ & $48(27 / 21)$ \\
Duration of hypertension (year) & $6 \pm 4$ & $5 \pm 4$ & $4 \pm 3$ & $6 \pm 5$ & $5 \pm 4$ \\
Doses at end study (ming) & 60 & 100 & 100 & 15 & 200 \\
Duration of treatment (weeks) & $29 \pm 11$ & $28 \pm 11$ & $26 \pm 10$ & $31 \pm 12$ & $27 \pm 10$ \\
\hline
\end{tabular}

